\title{
LOS NUEVOS DESARROLLOS TECNOLÓGICOS APLICADOS AL TRATAMIENTO PSICOLÓGICO
}

\author{
Wenceslao Peñate Castro $^{1 *}$, María José Roca Sánchez ${ }^{2}$, Tasmania Del Pino Sedeño ${ }^{1}$ \\ 'Dto. Psicología Clínica, Psicobiología y Metodología, Facultad de Psicología, Universidad de la Laguna \\ ${ }^{2}$ Instituto de Biotecnología - Universidad de Granada
}

Recibido, marzo 9/2014

Concepto de evaluación, abril 12/2014

Aceptado, junio 1/2014
Referencia: Peñate, W., Roca-Sánchez, M.J., \& Del Pino-Sedeño, T. (2014). Los nuevos desarrollos tecnológicos aplicados al tratamiento psicológico. Acta Colombiana de Psicología, 17 (2), pp. 91-101 DOI:10.14718/ACP.2014.17.2.10

Resumen

El desarrollo de nuevas tecnologías ha alcanzado prácticamente a todo el desarrollo humano. En el caso de la psicología clínica, ha significado nuevos avances en el conocimiento, evaluación y tratamientos psicológicos de diferentes problemas y trastornos. En este trabajo se revisan cuatro desarrollos que han tenido una expansión importante: el uso de internet para el tratamiento psicológico, el uso de la realidad virtual, el neurofeedback y la estimulación magnética transcraneal. Se presenta brevemente cada técnica o procedimiento, su aplicación a problemas concretos, sus ventajas y sus inconvenientes. Finalmente se discute el desarrollo de estos recursos y la necesidad de seguir aportando conocimientos que vayan mejorando su eficacia y su eficiencia, de acuerdo con tipos de pacientes, patologías, procedimiento específico, así como evitar efectos indeseables. Palabras clave: nuevas tecnologías, telesalud, realidad virtual, neurofeedback, estimulación transcraneal.

\section{NEW TECHNOLOGICAL DEVELOPMENTS APPLIED TO PSYCHOLOGICAL TREATMENT}

\begin{abstract}
The impact of new technologies has reached almost all human development. In the case of clinical psychology, has meant new advances in the understanding, assessment and treatment of various psychological problems and disorders. In this paper, four developments that have had a major expansion are reviewed: Internet-based psychological treatment, the use of virtual reality, neurofeedback, and transcranial magnetic stimulation. First, each technique or procedure is briefly presented. Then, its application to specific problems, its advantages and disadvantages are described. Finally, the development of these resources and the need to continue providing knowledge is discussed. These advances will improve their effectiveness and efficiency, according to types of patients, specific disorders, and to avoid side-effects.

Key words: new technologies, telehealth, virtual reality, neurofeedback, transcranial stimulation.
\end{abstract}

* Departamento de Psicología Clínica, Psicobiología y Metodología; Facultad de Psicología; Universidad de La Laguna; 38200 Universidad de La Laguna, Campus de Guajara. Tenerife. España, Tel.+34922317485 - Fax. Faz.+34922317461. wpenate@ull.es Este trabajo se ha realizado con el apoyo del proyecto PSI2013- 42912-R, financiado por el Ministerio de Economía y Competitividad del Gobierno de España 


\title{
OS NOVOS DESENVOLVIMENTOS TECNOLÓGICOS APLICADOS AO TRATAMENTO PSICOLÓGICO
}

\begin{abstract}
Resumo
O desenvolvimento de novas tecnologias alcançou praticamente a todo o desenvolvimento humano. No caso da psicologia clínica, significou novos avanços no conhecimento, avaliação e tratamentos psicológicos de diferentes problemas e transtornos. Neste trabalho são revisados quatro desenvolvimentos que tiveram uma expansão importante: o uso da internet para o tratamento psicológico, o uso da realidade virtual, ou neurofeedback e a estimulação magnética transcraniana. Cada técnica ou procedimento é brevemente apresentado, sua aplicação a problemas concretos, suas vantagens e seus inconvenientes. Finalmente discute-se o desenvolvimento destes recursos e a necessidade de seguir contribuindo com conhecimentos que possam melhorar sua eficácia e sua eficiência, de acordo com tipos de pacientes, patologias, procedimento específico, assim como evitar efeitos indesejáveis.

Palavras chave: novas tecnologias, telesaúde, realidade virtual, neurofeedback, estimulação transcraniana.
\end{abstract}

La psicología, como cualquier otra disciplina, ha mantenido una relación estrecha con los avances tecnológicos, tratando de utilizar esos avances en la mejora del desarrollo de la investigación y de sus aplicaciones prácticas. Muchos procedimientos en investigación básica y aplicada se han visto beneficiados del desarrollo de procedimientos de medición y evaluación más precisos y rigurosos (presentación de estímulos, medidas de tiempo de reacción, movimientos oculares, potenciales evocados, mapeo cerebral, entre otros), dando lugar no sólo a un conocimiento más cabal, sino a nuevos avances en el conocimiento (Norris, 2002).

Un aspecto importante de la psicología aplicada, la evaluación y el tratamiento de problemas mentales, también se ha visto beneficiada de estos avances, proporcionando en algunos casos nuevos modos de evaluación y tratamiento psicológico, así como nuevos desarrollos terapéuticos. Estos avances se han visto especialmente incrementados con la aparición de las tecnologías de la comunicación, siendo la telesalud/la telepsicología una de las más prometedoras líneas de investigación hoy día en la psicología clínica y de la salud. Sin embargo, no todas las aplicaciones clínicas de las nuevas tecnologías se agotan en la telepsicología, destacando también nuevas aplicaciones y nuevos desarrollos terapéuticos como el uso de la realidad virtual, la realidad aumentada, el uso de las presentaciones en $3 \mathrm{D}$, la estimulación magnética transcraneal o los nuevos desarrollos del biofeedback.

Lo que sigue a continuación es una ilustración de estos nuevos desarrollos dentro del ámbito de la psicología clínica, dirigiéndonos específicamente a los avances más destacados y con mayor atención en la investigación. En este sentido, revisaremos el uso de la telepsicología, el uso de la realidad virtual, el neurofeedback, y la estimulación magnética transcraneal.

\section{Telesalud y telepsicología}

La telesalud es el término que define el uso de las tecnologías de la información y la comunicación (TICs) para facilitar el acceso a la evaluación de la salud, el diagnóstico, la intervención, la consulta, la supervisión, la educación y la información a distancia (Landa-Durán, 2009; Nickelson, 1998; Peñate, 2012). El término telesalud incluye varios recursos como la telemedicina, la telepsiquiatría o la telepsicología. A continuación, se detallan algunas características de las tareas principales que componen la telepsicología: la evaluación, la psicoeducación, los programas de entrenamiento y el tratamiento psicológico.

El uso de internet como herramienta para la (i) evaluación psicológica ha crecido considerablemente. Actualmente, se encuentran multitud de sitios web que ofrecen evaluación psicológica online. Este procedimiento tiene ventajas claras. Sin embargo, la validez de las pruebas empleadas, la falta de control de la relación terapéutica y la ausencia de un proceso complejo de toma de decisión a la hora de emitir un diagnóstico puede facilitar la aparición de errores Tipo I y Tipo II. Las personas particularmente vulnerables pueden ser víctimas de la falta de información (falsos positivos) o la falta de claridad sobre el complejo proceso de toma de decisión en el diagnóstico.

Dentro de las páginas web relacionadas con la telepsicología, la (ii) psicoeducación representa el mayor volumen. En estas páginas se recoge información sobre diferentes patologías, sus características, curso y alternativas terapéuticas. A pesar de que desde un punto de vista meramente informativo, estas páginas pueden ser muy útiles, no existe evidencia a favor o en contra de este tipo de informaciones generales (Norris, 2002; Peñate, 2012). Además, la información sobre el curso, la gravedad y la cronicidad de la enfermedad puede tener un impacto negativo en la confianza y la seguridad de los pacientes (Peñate, 2012). 
Las páginas web sobre (iii) técnicas y procedimientos específicos para el entrenamiento conductual son también muy frecuentes. Este tipo de programas facilitan el entrenamiento sobre un sinfín de técnicas y recursos (relajación, respiración, mindfulness, autoinstrucciones, entre otros), que pueden facilitar el trabajo terapéutico. De nuevo, no hay datos que avalen estos recursos, pero los pacientes pueden utilizar estos entrenamientos por su cuenta y emplear el tiempo ahorrado en el desarrollo de la relación terapeutapaciente. Sin embargo, la calidad y el enfoque de estos recursos es muy diversa, lo que puede confundir e incluso perjudicar a los pacientes.

Con todo, lo que mayor interés ha despertado en el uso de la telesalud asociado a la psicología son los aplicativos diseñados para facilitar el (iv) tratamiento psicológico. Por su importancia, lo desarrollamos a continuación.

\section{Tratamiento psicológico vía internet}

El tratamiento psicológico por internet representa una nueva forma más rápida y flexible de los denominados tratamientos basados en el manual, cuya eficacia se ha demostrado empíricamente (Wilson, 1996). En general, estos tratamientos incluyen componentes ya comentados relativos a psicoeducación sobre el trastorno objetivo del tratamiento, la evaluación psicológica y los componentes de la terapia (con una secuencia predeterminada).

Aunque los programas por internet tienen un diseño muy claro, son muy diversos (Nelson, Bui \& Velásquez, 2011; Titov, 2007). Esta diversidad se puede concretar en los siguientes aspectos: la duración del tratamiento (que varía de 10-15 minutos a más de 10 semanas); la presentación (que aunque normalmente es a través de audio y texto, puede incluir imágenes y videos); la prestación del servicio (que oscila desde una estrategia interactiva online a un recurso con acceso regulado, en ocasiones sujeto a una tarifa); los comentarios del terapeuta (que se pueden presentar en línea o a través de correo electrónico); el acceso al contenido del programa y la evaluación (con acceso libre a todos los recursos frente a un acceso limitado, en función de las características del paciente y que en su versión más sofisticada se presenta en forma de árboles de decisión); la aplicación del refuerzo social (inmediato o demorado a través de correo electrónico); la disponibilidad de mensajes estratégicos adaptados a cada paciente (algunos programas analizan la salud y las conductas de riesgo de cada paciente); y la etapa de cambio (motivación) para decidir cuál es la ruta a seguir en la ejecución del programa.

\section{Efectividad de los tratamientos por internet}

El crecimiento de los tratamientos psicológicos vía internet no se ha visto acompañado por ensayos clínicos que demuestren su eficacia. El hecho de que estos recursos se basen en programas validados empíricamente no es razón suficiente para garantizar su validez (Frueh, Monnier, Elhai, Grubaugh y Knapp, 2004). Además, es difícil comparar los estudios debido a las diferencias entre ellos: en las muestras, los trastornos abordados, el contenido y la duración de la terapia, el acceso al terapeuta, etc. Aun así, en los últimos años se han realizado varias revisiones y meta-análisis sobre la eficacia y la eficiencia de estos programas (Andersson y Cuijpers, 2009; Andrews, Cuijpers, Craske, McEvoy y Titov, 2010; Newman, Szkodny, Llera y Przeworski, 2011). El análisis de estas revisiones sugiere que la eficacia es mayor: a) En los programas más extensos. b) Con niveles leves a moderados de trastorno. c) En pacientes más motivados. d) En función del contacto con el terapeuta, cuando el paciente puede tener algún tipo de contacto con el terapeuta y recibir retroalimentación y seguimiento del programa. e) En programas para el tratamiento de la ansiedad, las fobias y la depresión.

\section{Ventajas y desventajas de los tratamientos por internet}

De acuerdo con las revisiones sistemáticas y meta-análisis realizados ya citados (Andersson \& Cuijpers, 2009; Andrews et al., 2010; Newman et al., 2011), se pueden entresacar las siguientes ventajas y desventajas de los tratamientos vía internet.

La primera ventaja es que libera al paciente de la estigmatización y le da la oportunidad de mantener en privado su tratamiento. Una segunda ventaja está vinculada a la movilidad: las personas con movilidad reducida o alejadas geográficamente pueden encontrar en los tratamientos por internet una buena alternativa. La tercera está vinculada a las dificultades inherentes a los síntomas de una enfermedad o un trastorno, como la agorafobia, que lleva a los pacientes a permanecer habitualmente en sus casas. La cuarta se relaciona con la flexibilidad en el acceso y el tiempo, permitiendo que se pueda acceder a cualquier hora, en función de la disponibilidad de cada uno. Por último, una ventaja adicional es económica, el coste de estos programas suele ser más bajo que el de los tradicionales (Andersson \& Cuijpers, 2009; Andrews et al., 2010; Newman et al., 2011).

Por otro lado, también esas revisiones y meta-análisis han identificado desventajas. Éstas se pueden resumir en que no todos los programas siguen un procedimiento riguroso y empíricamente validado (Andersson \& Cuijpers, 2009), el acceso de los pacientes al programa no siempre se controla y no suele haber control sobre la fiabilidad de las respuestas de los pacientes. No siempre hay control sobre el trabajo terapéutico (tareas, autorregistros ), lo que significa que no hay un control real sobre la adhesión al tratamiento (Andersson \& Cuijpers, 2009; Newman et 
al., 2011). No todos los programas incluyen mecanismos mediante los cuales el paciente puede ponerse en contacto con el terapeuta y aclarar dudas. Pocos estudios incluyen evaluación postratamiento para garantizar la seguridad de la información obtenida. Finalmente, no es usual que se disponga de datos de seguimiento, por lo que la información sobre la consolidación de las ganancias se ve limitada (Newman et al., 2011).

\section{El uso de la realidad virtual}

La realidad virtual (RV) consiste en una realidad creada (modelada) a partir de escenarios reales. La creación de estos escenarios permite diferentes opciones y alternativas, a partir de la movilidad que el participante tiene sobre el escenario. La realidad virtual puede ser complementada con otras fuentes de información como puede ser la auditiva o la táctil, pero es la visual la que predomina, lógicamente (Vanni et al., 2013).

Un prerrequisito de un escenario virtual es su capacidad inmersiva: en qué medida existe una sensación de presencia real en el escenario creado, en qué medida se acerca a la situación real. La presentación estereoscópica es una de las mejores garantías para esa sensación de presencia, de realidad de los escenarios.

Existen dos grandes modalidades de presentación de la RV: la head mounted device (HMD, por sus siglas en inglés) y la Cave Automatic Virtual Environment (CAVE, por sus siglas en inglés). La HMD consiste en un casco/ gafas que presenta la información para cada campo visual. Estas gafas están conectadas a un ordenador. En el caso de la modalidad CAVE, los entornos virtuales son proyectados sobre una pantalla. Esta presentación se puede hacer en una pantalla que genera una sensación de 3D o una pantalla plana, con los escenarios en 3D (en este caso se necesitan gafas para 3D).

Una versión del uso de la RV como estrategia expositiva para las fobias lo representa la realidad aumentada (RA). Esta estrategia combina elementos reales con elementos virtuales, lo que permite introducir en el entorno habitual del paciente los estímulos fóbicos, complementando la realidad en lugar de reemplazarla. Los elementos virtuales son introducidos en tiempo real, por lo que el paciente puede sentir en su entorno aquellos elementos temidos. El desarrollo técnico es el que mejor puede garantizar que existan las menores discrepancias entre medio real y los estímulos virtuales, siendo éste un aspecto fundamental para la credibilidad del procedimiento.

La propuesta de utilizar tecnologías de realidad virtual para el tratamiento de los trastornos psicológicos fue llevada a cabo por primera vez en el Human-Computer Interaction
Group de la Universidad Clark de Atlanta (North \& North, 1994), donde acuñaron el término "Terapia de Exposición a Realidad Virtual" (TERV), tratando con esta técnica un caso de fobia a volar. Los buenos resultados favorecieron que investigaciones posteriores ampliaran el campo de aplicación de la RV a distintos ámbitos (i.e., Carpio et al., 2008; Córdoba \& Larreamendy-Joerns, 2007; Montes González \& Ochoa Angrino, 2006; Ossa, 2011) aunque ha sido en los trastornos fóbicos en los que se han aplicado con mayor frecuencia, incluyendo una fobia tan compleja como la agorafobia (i.e., González-Lorenzo et al., 2011).

\section{Efectividad de la realidad virtual}

De los meta-análisis y las revisiones sistemáticas realizadas (Gonçalves, Pedrozo, Coutinho, Figueira \& Ventura, 2012; Meyerbroeker, Morina, Kerkhof \& Emmelkamp, 2013; Opriş et al., 2012; Vanni et al., 2013) se puede constatar que el uso de RV es muy eficaz (con tamaños del efecto elevados), sobre todo en el tratamiento de fobias específicas y agorafobia, en comparación con grupos tratados con terapias tradicionales eficaces, entre ellas la exposición en vivo (Peñate et al., 2014). Enlarevisión de Meyerbröker \& Emmelkamp (2010), se postula que esta eficacia puede estar mediada porque produce ciertos cambios a nivel cognitivo, representados por un aumento en la autoeficacia y en las autoinstrucciones que generan durante la exposición a los estímulos fóbicos.

Ventajas y desventajas de los tratamientos que utilizan la $R V$

Una de las mayores ventajas del uso de la realidad virtual durante la terapia de los trastornos de ansiedad parece ser que implica una mayor adhesión al tratamiento (Peñate et al., 2014). Además, en algunos pacientes reacios a enfrentarse a estímulos reales, la exposición con RV podría jugar un rol intermedio, enfrentándose con su contraparte virtual (Shiban, Pauli \& Mühlberger, 2013). Otra ventaja del uso de RV es la prevención de eventos incontrolables, al ser un ambiente regulado, disminuyendo la posibilidad de que aparezcan hechos imprevistos (Meyerbroeker et al., 2013; Obando, Villalobos $\&$ Arango, 2010). Una cuarta ventaja es el mayor control de los estímulos por parte del terapeuta, lo que conlleva un mayor control de las sesiones de exposición (sobre todo en la RA). Una quinta ventaja es la individualización de las sesiones de exposición para cada paciente, así como la posibilidad de que terapeuta y paciente puedan compartir la experiencia de la exposición. En sexto lugar, la RV facilita la evocación de recuerdos que al paciente le puede resultar difícil rememorar, al complementar las imágenes mentales del paciente con pistas sensoriales.

La mayor y más importante desventaja en el uso de la RV para el tratamiento clínico de pacientes es el elevado coste, 
tanto de los aparatos, como el desarrollo de los escenarios virtuales (software). Una segunda desventaja es el hecho de que trabajar con tecnología informática conlleva el riesgo de fallos en el sistema. Condicionado a esta dependencia técnica, está la capacidad inmersiva de los escenarios virtuales, que necesitan ser "creíbles" para los pacientes. Una tercera desventaja es que la experiencia de RV puede distraer a los pacientes de sus emociones y centrarlos en la experiencia tecnológica en sí misma, convirtiéndose en un método de evitación en la práctica. Finalmente, desde un punto de vista científico, se necesitan más estudios que incluyan pruebas de oro (exponer al paciente a los estímulos reales y observar si finalmente son capaces de permanecer ante ellos), y estudios con seguimientos que indiquen en qué medida se consolidan los resultados.

\section{El uso del neurofeedback}

La técnica de biofeedback es un método tradicional de tratamiento durante el cual los pacientes toman conciencia y aprenden a controlar su propia fisiología, con el objetivo de mejorar tanto su salud física como su salud psicológica. El neurofeedback (NF, por sus siglas en inglés), es una subespecialización del biofeedback, también llamado biofeedback EEG. Utiliza la electro-encefalografía (EEG) del paciente como retroalimentación para modificar los patrones de actividad eléctrica cerebral (Larsen \& Sherlin, 2013), sin la introducción de actividad eléctrica o magnética, o compuestos farmacológicos, evitando por lo tanto, que el cerebro se vuelva dependiente de influencias externas para mejorar su funcionamiento (Niv, 2013). La actividad cerebral del paciente se mide con un electrodo que incorpora un código de letras y números para identificar el lóbulo y la ubicación del hemisferio, respectivamente. Los mecanismos por los cuales funciona se suelen vincular a los mecanismos clásicos del condicionamiento operante de aprendizaje que entrenan el cerebro para mejorar la regulación del mismo, proporcionándole información (visual, auditiva y/o táctil) en tiempo real (Lofthouse, Arnold, Hersch, Hurt \& DeBeus, 2012).

El origen del NF está directamente relacionado con la primera demostración de condicionamiento operante EEG. Sterman y colaboradores condujeron una serie de estudios en el contexto de la investigación sobre el sueño, en los que investigaban la supresión aprendida de una respuesta de presión de palanca en gatos recompensada de manera previa con comida (Roth, Sterman \& Clemente, 1967). Los investigadores observaron un ritmo electro-encefalográfico sobre la corteza motora con una frecuencia específica. $\mathrm{Al}$ entrenar a los gatos para producirlo voluntariamente, mediante la contingencia con una recompensa comestible, consiguieron la autorregulación del EEG (Sterman \& Egner, 2006). Posteriormente, esta investigación ha sido extrapolada con éxito a los seres humanos, modificándose determinados patrones cerebrales y sus correlatos comportamentales (Sterman \& Egner, 2006).

Durante el entrenamiento en NF, el paciente está sentado visualizando un ordenador (se pueden utilizar juegos, rompecabezas, secuencias de imágenes, etc.). Es importante que el paciente esté motivado para ver la tarea, así como que perciba los objetivos de la retroalimentación como ejercicios especiales para el cerebro (Sterman \& Egner, 2006). El objetivo del tratamiento con NF consiste en seleccionar la actividad cerebral que queremos alterar, dependiendo de los síntomas del paciente y de su patrón de actividad cerebral. Se espera que cambiando la actividad cerebral y el funcionamiento cognitivo del paciente cambie el comportamiento (Breteler, Pesch, Nadorp, Best \&Tomasoa, 2012). En definitiva, podríamos decir que el NF identifica patrones anormales detectados en el EEG y los modifica mediante condicionamiento operante (Mustaca, 2003).

\section{Efectividad del neurofeedback}

De acuerdo con algunas revisiones y metanálisis (Dias \& van Deusen, 2011; Gevensleben, Rothenberger, Moll \& Heinrich, 2012; Larsen \& Sherlin, 2013; Lofthouse et al., 2012; Moriyama et al., 2012), el NF parece ser eficaz en el tratamiento de TDAH, epilepsia, y en trastornos de ansiedad. También se han aportado datos sobre la eficacia en lesiones cerebrales traumáticas, alcoholismo/abuso de sustancias e insomnio. Donde la evidencia de su eficacia es insuficiente ha sido en los trastornos depresivos, el trastorno por estrés postraumático, tinitus y autismo (Coben, Linden \& Myers, 2010; Holtmann et al., 2011). A pesar de estos datos, existen problemas metodológicos, necesitándose más investigación que compare el NF con controles placebo y otros tipos de tratamientos (Loo \& Makeig, 2012). En cuanto a su efectividad a largo plazo, muy pocos estudios la han examinado, pero los que sí lo han hecho encuentran resultados prometedores (Moriyama et al., 2012).

\section{Ventajas y desventajas en el uso del Neurofeedback}

Varias son las ventajas en el uso de NF: a) altera la actividad cerebral sin la introducción de influencias externas para mejorar su funcionamiento (Larsen \& Sherlin, 2013). b) Este entrenamiento se realiza utilizando un videojuego, lo cual lo hace más motivante (Sterman \& Egner, 2006). c) Reduce el uso de tratamiento farmacológico, disminuyendo la dependencia del paciente de una sustancia externa (Moriyama et al., 2012). d) No se han encontrado efectos secundarios (Moriyama et al., 2012). 
Las desventajas se han situado en que es una técnica que requiere de una motivación y una atención especial por parte del paciente (Sterman \& Egner, 2006). El entrenamiento puede volverse laborioso, ya que se necesitan varias sesiones para que empiece a ser efectivo (Larsen \& Sherlin, 2013). Además, requiere de un personal clínico especializado, entrenado en el manejo de la técnica (Sterman \& Egner, 2006). Por último, y no menos importante, los aparatos necesarios para ejecutar el NF son costosos (hoy día). (Sterman \& Egner, 2006).

\section{El uso de la estimulación magnética transcraneal}

La estimulación magnética transcraneal (EMT), introducida por Anthony Barker en 1985 en el estudio de las neurociencias cognitivas (Barker, Jalinous \& Freeston, 1985) facilita la estimulación del tejido nervioso (córtex, médula espinal y nervios periféricos) de manera no invasiva e indolora, permitiendo de esta manera interferir en la actividad normal del cerebro. Funciona a través de campos magnéticos oscilantes administrados por una bobina situada sobre el cuero cabelludo. Este campo de inducción electromagnética, cuando alcanza la magnitud y densidad suficientes puede llegar a despolarizar las neuronas, y cuando se aplica de manera repetitiva, puede modular la excitabilidad cortical, disminuyéndola o incrementándola, incluso una vez finalizada la estimulación, lo que tiene consecuencias tanto en el comportamiento como en el potencial terapéutico (Rossi, Hallett, Rossini \& Pascual-Leone, 2009).

La EMT se clasifica en simple, apareada o repetitiva (EMTr) según sea la frecuencia de estimulación. La EMT simple consiste en un pulso de una frecuencia inferior a 1 $\mathrm{Hz}$ que se administra en un momento y con una duración precisa, cuenta con una buena resolución temporal y puede despolarizar neuronas en el córtex motor y evocar efectos medibles en el músculo contralateral. Por su parte, la EMT con pulsos apareados consiste en aplicar dos pulsos separados por un pequeño intervalo de tiempo (entre 1 y $20 \mathrm{~ms}$ ) con el objetivo de explorar la función de las interneuronas inhibitorias intracorticales. Tanto la inhibición como la facilitación intracortical han sido estudiadas por este método; los pulsos pueden producirse a intervalos variables en la misma o en distintas áreas del cerebro (Fernández del Olmo \& Cudeiro Mazaira, 2004). La EMT con pulsos apareados ha sido usada en el estudio de la patofisiología de varios trastornos neurológicos y psiquiátricos (Kobayashi \& Pascual-Leone, 2003). Por último, en la EMT repetitiva (EMTr) se aplican trenes de pulsos con una frecuencia de hasta $50 \mathrm{~Hz}$ durante decenas, centenas o miles de milisegundos; se divide entre EMTr de baja y de alta frecuencia. La EMTr puede modificar la excitabilidad de la corteza cerebral, así como en zonas remotas a través de conexiones anatómicas funcionales. También se ha visto que es útil a la hora de localizar qué áreas cerebrales interfieren en una función (Kobayashi \& Pascual-Leone, 2003).

\section{Efectividad de la EMT}

Según aportan los meta-análisis y revisiones (Aleman, 2013; Berlim, Neufeld \& Van den Eynde, 2013; Berlim, van den Eynde, Tovar-Perdomo \& Daskalakis, 2014; Leon-Sarmiento, Granadillo \& Bayona, 2013; Theodoroff $\&$ Folmer, 2013), la EMT es una herramienta de un gran potencial en diversas patologías como la depresión, especialmente en la depresión resistente al tratamiento (PérezWehbe, Perestelo-Pérez, Bethencourt-Pérez, Cuellar-Pompa $\&$ Peñate, 2014), y, en menor medida, en las alucinaciones típicas de la esquizofrenia, TDAH, trastornos del espectro autista y apnea del sueño. Pocos autores recomiendan su uso en el TOC y en el estrés postraumático, siendo necesaria más experimentación que clarifique su uso.

$\mathrm{Su}$ administración es segura y cuenta con pocos casos de convulsiones, asociadas éstas principalmente con su uso en frecuencias altas ( $10 \mathrm{~Hz}$ o por encima) (Fuggetta \& Noh, 2013; Rossi et al., 2009). El meta-análisis realizado por Janicak et al. (2008), evaluó la seguridad de la estimulación magnética transcraneal repetitiva y la eficacia y duración de su beneficio. El resultado es que la EMTr fue bien tolerada y se asocia con una baja incidencia de efectos secundarios que fueron en su mayoría de leves a moderados.

En cuanto a los aspectos metodológicos, se pone de manifiesto la necesidad de realizar una reunificación de criterios en cuanto a la selección de pacientes, mayores tamaños muestrales, eliminar sesgos relacionados con la edad, el consumo de medicamentos, y el estado cognitivo $\mathrm{y}$ afectivo de los pacientes.

\section{Ventajas y desventajas de la EMT}

La mayor ventaja de la EMT es su utilización en trastornos para los que los recursos terapéuticos existentes son muy pobres, permitiendo reducir el tratamiento farmacológico (Aleman, 2013). Una segunda ventaja, es que su uso conlleva asociado un aumento del conocimiento de cómo funciona el sistema nervioso, pudiendo utilizar, por ejemplo, la técnica en entrenamiento para el conocimiento de las adaptaciones neuronales a diferentes tipos de actividades (Leon-Sarmiento et al. 2013). Una tercera ventaja está relacionada con ciertas propiedades anticonvulsivas, por lo que en pacientes con riesgo de crisis epilépticas parece ser el tratamiento de elección (Berlim, Van den Eynde y Daskalakis, 2013).

Entre sus desventajas se han descrito en algunos casos cefaleas de tipo tensional que son fácilmente tratables con analgésicos convencionales (Aleman, 2013). Una segunda desventaja está relacionada con el riesgo de desencadenar 
convulsiones al aplicar la EMT a alta intensidad (LeonSarmiento et al., 2013). Otra de las desventajas de esta técnica, común al resto de las técnicas mencionadas en este artículo, es el elevado costo económico del equipo, así como la formación del personal encargado de administrar las sesiones.

\section{DISCUSIÓN}

En la tabla 1, se resumen las principales características de los nuevos desarrollos comentados hasta aquí, sus aplicaciones más relevantes y sus ventajas e inconvenientes más destacados.

Un aspecto que distingue a estos procedimientos es que tratan de proporcionar ventajas frente a los procedimientos tradicionales, ya sea porque suponen una nueva técnica en el tratamiento de los trastornos psicológicos, o porque son una versión tecnológica de materiales usualmente utilizados en las terapias tradicionales, o porque constituyen una modificación en la forma de brindar el tratamiento, o porque, incluso, la aportación pueda significar un modo más sencillo o viable de llevar a cabo el tratamiento psicológico.

En este sentido, estos nuevos desarrollos poseen una serie de ventajas (en comparación con la práctica tradicional), no sólo porque eliminan aquellas dificultades a las que un paciente se podría enfrentar a la hora de acceder a un profesional, (como en el caso de los tratamientos por internet), sino también porque facilitan el tránsito de un paciente a una parte de la terapia, que por la dificultad que ésta conlleva (p.e., la exposición en vivo), supone en muchos casos, el abandono de la terapia (RV, Peñate et al., 2014). Además, algunas de estas nuevas técnicas nos están

Tabla 1.

Resumen de las características de los nuevos desarrollos tecnológicos en el tratamiento psicológico.

\begin{tabular}{|c|c|c|c|c|}
\hline Procedimiento & Características & Aplicaciones & Ventajas & Inconvenientes \\
\hline Telepsicología & $\begin{array}{l}\text { Tratamiento } \\
\text { psicológico vía } \\
\text { internet. }\end{array}$ & $\begin{array}{l}\text { Diferentes trastornos/ } \\
\text { problemas } \\
\text { psicológicos. }\end{array}$ & $\begin{array}{l}\text { Menor estigmatización. } \\
\text { Reduce los problemas de movilidad. } \\
\text { Versatilidad en la accesibilidad. } \\
\text { Económico. } \\
\text { Patologías que impiden acudir a un } \\
\text { servicio (Andersson \& Cuijpers, } \\
\text { 2009; Andrews et al., 2010; Newman } \\
\text { et al., 2011). }\end{array}$ & $\begin{array}{l}\text { No siempre programas rigurosos. } \\
\text { No control sobre el trabajo terapéutico. } \\
\text { Necesidad de algún contacto con } \\
\text { terapeuta (Andersson \& Cuijpers, } \\
\text { 2009; Andrews et al., 2010; Newman } \\
\text { et al., 2011). }\end{array}$ \\
\hline $\begin{array}{l}\text { Realidad } \\
\text { virtual }\end{array}$ & $\begin{array}{l}\text { Simulación } \\
\text { de contextos } \\
\text { y condiciones } \\
\text { en relación a } \\
\text { los trastornos } \\
\text { psicológicos. }\end{array}$ & $\begin{array}{l}\text { Diversos trastornos } \\
\text { (estrés post- } \\
\text { traumático, trastornos } \\
\text { alimentarios, pánico, } \\
\text { etc.). } \\
\text { Especialmente en } \\
\text { fobias. }\end{array}$ & $\begin{array}{l}\text { Mayor adherencia en pacientes } \\
\text { crónicos. } \\
\text { Disminución de incertidumbres. } \\
\text { Mayor control del terapeuta. } \\
\text { Control sobre la exposición a } \\
\text { estímulos temidos (Gonçalves et al., } \\
\text { 2012; Meyerbroeker et al., 2013; } \\
\text { Opriş et al., 2012; Vanni et al., } \\
\text { 2013). }\end{array}$ & $\begin{array}{l}\text { Representatividad de los escenarios. } \\
\text { Capacidad inmersiva. } \\
\text { Se puede atender más a la tecnología } \\
\text { que al proceso terapéutico. } \\
\text { Necesidad de pruebas de oro } \\
\text { (Gonçalves et al., 2012; Meyerbroeker } \\
\text { et al., 2013; Opriş et al., 2012; Vanni et } \\
\text { al., 2013). }\end{array}$ \\
\hline Neurofeedback & $\begin{array}{l}\text { Un modo de } \\
\text { biofeedback. Uso } \\
\text { de la EEG como } \\
\text { retroalimentación } \\
\text { para modificar } \\
\text { los patrones de } \\
\text { actividad cerebral. }\end{array}$ & $\begin{array}{l}\text { TDAH, epilepsia, } \\
\text { trastornos de ansiedad. } \\
\text { También alcoholismo/ } \\
\text { abuso de sustancias e } \\
\text { insomnio. }\end{array}$ & $\begin{array}{l}\text { No efectos secundarios (Moriyama } \\
\text { et al., 2012). } \\
\text { No necesita de fármacos para alterar } \\
\text { la EEG (Larsen \& Sherlin, 2013). } \\
\text { Motivante (Sterman \& Egner, 2006). }\end{array}$ & $\begin{array}{l}\text { Atención especial por el paciente } \\
\text { (Sterman \& Egner, 2006). . } \\
\text { Entrenamiento prolongado (Larsen \& } \\
\text { Sherlin, 2013). } \\
\text { Personal entrenado (Sterman \& Egner, } \\
\text { 2006). } \\
\text { No económico (Sterman \& Egner, } \\
\text { 2006). }\end{array}$ \\
\hline $\begin{array}{l}\text { Estimulación } \\
\text { magnética } \\
\text { transcraneal }\end{array}$ & $\begin{array}{l}\text { Inhibición de } \\
\text { determinada } \\
\text { actividad cerebral } \\
\text { por medio de } \\
\text { la estimulación } \\
\text { electromagnética } \\
\text { externa. }\end{array}$ & $\begin{array}{l}\text { Depresión refractaria. } \\
\text { Alucinaciones. } \\
\text { TDAH. } \\
\text { Trastorno del espectro } \\
\text { autista. } \\
\text { Apnea del sueño. }\end{array}$ & $\begin{array}{l}\text { (Schutter, 2009). } \\
\text { Especial para algunas patologías } \\
\text { resistentes (Aleman, 2013; Pérez- } \\
\text { Wehbe et al., 2014)). }\end{array}$ & $\begin{array}{l}\text { Algunos efectos secundarios (cefaleas, } \\
\text { convulsiones...) (Aleman, 2013). } \\
\text { Personal especializado. } \\
\text { No económico. }\end{array}$ \\
\hline
\end{tabular}


ayudando a comprender mejor cómo funciona el cerebro y cómo se relaciona con la conducta humana (NF, Niv, 2013). De manera que cada vez nos acercamos a técnicas más precisas, focalizadas y menos invasivas que ayuden al paciente a ir reduciendo el consumo de fármacos y con ello, también los efectos secundarios indeseables de éstos (EMT Aleman, 2013), convirtiéndose así en una alternativa terapéutica para algunos problemas que no cuentan hoy en día con tratamientos eficientes.

Los cuatro desarrollos comentados se orientan de manera distintas dentro del ámbito de los tratamientos psicológicos, representando cada uno ciertos avances en relación con los procedimientos tradicionales de tratamiento. En este sentido, los tratamientos psicológicos servidos vía internet, representan la novedad más importante en el formato de presentación, siendo, como se ha comentado, una versión actual de los tratamientos basados en el manual. Pero el mero hecho de su presentación vía internet, con la posibilidad de usar al mismo tiempo distintos soportes (audio, video, texto ), y sus posibilidades interactivas, implican un avance en relación con las biblioterapias tradicionales. Sin embargo, también puede ocurrir que los usuarios vean estos tratamientos como "nuevos principios activos" que, evidentemente, no poseen, porque se fundamentan en tratamientos ya validados en su presentación tradicional. Si a esto se añade que algunos programas no están apoyados empíricamente, lo que era una ventaja evidente puede transformarse en un serio inconveniente, bien sea porque no va cumplir con las expectativas y deseos de los pacientes, o bien, porque el propio procedimiento adolece de una falta de apoyo en la investigación (Frueh et al., 2004).

El uso de la RV ha sido muy versátil, pero su mayor utilización ha sido como un recurso expositivo para las fobias y para problemas donde se necesite "exponer" al paciente a una serie de estímulos. Como hemos comentado, la RV ha significado una mayor adherencia a los tratamientos, lo que es realmente relevante en pacientes refractarios, que ya han pasado por varios tratamientos sin éxito (Peñate et al., 2014). Sin embargo, de nuevo, se pueden estar creando falsas expectativas, con posibles efectos placebos, que se pierden con el paso del tiempo. Además, la no presencia en las investigaciones de pruebas de oro (enfrentar al paciente a la situación real), puede estar creando una suerte de mundo en paralelo (más seguro que el mundo real) donde el paciente es capaz de enfrentar sus problemas de manera 'virtual', pero con graves problemas de generalización.

De alguna manera el neurofeedback sería, como la telesalud, una variante en la presentación de una técnica tradicional como es biofeedback. Sin embargo, el hecho de que suponga la posibilidad de condicionar respuestas cerebrales ya en sí mismo supone un cambio conceptual y no (solamente) formal. Lo que sí parece claro es que es un avance técnico que hay que integrar dentro de un marco más amplio de tratamiento, para que resultados tan relevantes con patologías tan resistentes como el abuso de sustancias, síntomas de la epilepsia y TDAH no sean meros espejismos y puedan consolidarse (Berlim et al., 2014).

Algo similar se puede comentar del uso de la EMT. Son resultados prometedores, especialmente con patologías tan complicadas como la depresión refractaria. Sin embargo, los cambios tienen un alcance limitado y seguramente serán necesarios otros apoyos terapéuticos, que fortalezcan las mejoras (p.e., Pérez-Wehbe et al., 2014). En ambos casos (NF y EMT) son productos sanitarios para abordar problemas de índole psicológica, donde hay una persona que percibe y analiza la realidad, incluyendo el propio proceso terapéutico, por lo que ese soporte mayor parece necesario, no desestimando la importancia de la relación terapeuta-paciente (Klein et al., 2003).

En definitiva, estos cuatro desarrollos ejemplifican nuevas alternativas terapéuticas que están teniendo un auge destacable, con las consiguientes expectativas despertadas en pacientes, que ven en ellas una esperanza de que por fin se pueda abordar con éxito su sufrimiento. Sin embargo, quedan por establecer y aclarar muchos aspectos de estas técnicas. Por ejemplo, falta definir su eficacia y efectividad de acuerdo a los tipos de patología, el modo en que se presta el tratamiento y el control riguroso sobre el mismo.

Sin ello, esos efectos pueden no ser más que un caso más del "veredicto del pájaro dodo" (Luyten, Blatt, Van Houdenhove \& Corveleyn, 2006), donde cualquier innovación bien intencionada va a tener un éxito inmediato, pero que se diluye con el paso del tiempo.

\section{REFERENCIAS}

Aleman, A. (2013). Use of Repetitive Transcranial Magnetic Stimulation for Treatment in Psychiatry. Clinical Psychopharmacology and Neuroscience: The Official Scientific Journal of the Korean College of Neuropsychopharmacology, 11(2), 53-59. doi: 10.9758/cpn.2013.11.2.53

Andersson, G., \& Cuijpers, P. (2009). Internet-based and other computerized psychological treatments for adult depression. Cognitive Behaviour Therapy, 38, 196-205. doi: 10.1080 /16506070903318960

Andrews, G., Cuijpers, P., Craske, M. G., McEvoy, P., \& Titov, N. (2010). Computer therapy for the anxiety and depressive disorders is effective, acceptable and practical health care: a meta-analysis. PloS One, 5(10), e13196. doi: 10.1371/journal.pone.0013196 
Barker, A. T., Jalinous, R., \& Freeston, I. L. (1985). Non-invasive magnetic stimulation of human motor cortex. Lancet, 1, 1106-1107.

Berlim, M. T., Neufeld, N. H., \& Van den Eynde, F. (2013). Repetitive transcranial magnetic stimulation (rTMS) for obsessive-compulsive disorder (OCD): an exploratory metaanalysis of randomized and sham-controlled trials. Journal of Psychiatric Research, 47(8), 999-1006. doi: 10.1016/j. jpsychires.2013.03.022

Berlim, M. T., Van den Eynde, F., \& Daskalakis, Z. J. (2013). Clinically meaningful efficacy and acceptability of low-frequency repetitive transcranial magnetic stimulation (rTMS) for treating primary major depression: a meta-analysis of randomized, double-blind and sham-controlled trials. Neuropsychopharmacology: Official Publication of the American College of Neuropsychopharmacology, 38(4), 543-51. doi: 10.1038/npp.2012.237

Berlim, M. T., van den Eynde, F., Tovar-Perdomo, S., \& Daskalakis, Z. J. (2014). Response, remission and drop-out rates following high-frequency repetitive transcranial magnetic stimulation (rTMS) for treating major depression: a systematic review and meta-analysis of randomized, double-blind and sham-controlled trials. Psychological Medicine, 44, 1-15. doi: b10.1017/S0033291713000512

Breteler, R., Pesch, W., Nadorp, M., Best, N., \& Tomasoa, X. (2012). Neurofeedback in Residential Children and Adolescents with Mild Mental Retardation and ADHD Behavior. Journal of Neurotherapy, 16(3), 172-182. doi: 10.1080/10874208.2012.705742

Carpio, C., Silva, H., Reyes, A., Pacheco-Lechón, L., Morales, G., Arroyo, R., Canales, C., \& Pacheco, V. (2008). Factores lingüísticos y consecuencias situacionales en la elección de estudiantes universitarios entre colaborar y no colaborar en tareas académicas: un análisis experimental. Acta Colombiana de Psicología, 11(2), 115-126. Recuperado de http://www.scielo.org.co/scielo.php?script=sci_arttext\&pid $=\mathrm{S} 0123-91552008000200011$

Coben, R., Linden, M., \& Myers, T. E. (2010). Neurofeedback for autistic spectrum disorder: a review of the literature. Applied Psychophysiology and Biofeedback, 35(1), 83105. doi: 10.1007/s10484-009-9117-y

Córdoba, M., \& Larreamendy-Joerns, J. (2007). Variación en estilos de experimentación y conocimiento específico. Acta Colombiana de Psicología, 10(1), 25-34. Recuperado de http://portalweb.ucatolica.edu.co/easyWeb2/acta/pdfs/ v10n1/Acta10v1Art3.pdf

Dias, A. M., \& van Deusen, A. (2011). A new neurofeedback protocol for depression The Spanish Journal of Psychology, 14(1), 374-84.

Fernández del Olmo, M., \& Cudeiro Mazaira, J. (2004). Estimulación magnética transcraneal: Principios básicos y aplicaciones en la actividad física y el deporte. Motricidad. European Journal of Human Movement, 12, 7-20.
Frueh, B. C., Monnier, J., Elhai, J. D., Grubaugh, A. L., \& Knapp, R. G. (2004). Telepsychiatry treatment outcome research methodology: efficacy versus effectiveness. Telemedicine Journal and E-Health: The Official Journal of the American Telemedicine Association, 10(4), 455-458. doi: 10.1089/tmj.2004.10.455

Fuggetta, G., \& Noh, N. A. (2013). A neurophysiological insight into the potential link between transcranial magnetic stimulation, thalamocortical dysrhythmia and neuropsychiatric disorders. Experimental Neurology, 245, 87-95. doi: 10.1016/j.expneurol.2012.10.010

Gevensleben, H., Rothenberger, A., Moll, G. H., \& Heinrich, H. (2012). Neurofeedback in children with ADHD: validation and challenges. Expert Review of Neurotherapeutics, 12(4), 447-60. doi: 10.1586/ern.12.22

Gonçalves, R., Pedrozo, A. L., Coutinho, E. S. F., Figueira, I., \& Ventura, P. (2012). Efficacy of virtual reality exposure therapy in the treatment of PTSD: a systematic review. PloS One, 7(12), e48469. doi: 10.1371/journal.pone.0048469

Holtmann, M., Steiner, S., Hohmann, S., Poustka, L., Banaschewski, T., \& Bölte, S. (2011). Neurofeedback in autism spectrum disorders. Developmental Medicine and Child Neurology, 53(11), 986-93. doi: 10.1111/j.1469-8749.2011.04043.x

Janicak, P. G., O’Reardon, J. P., Sampson, S. M., Husain, M. M., Lisanby, S. H., Rado, J. T., ... Demitrack, M. A. (2008). Transcranial magnetic stimulation in the treatment of major depressive disorder: a comprehensive summary of safety experience from acute exposure, extended exposure, and during reintroduction treatment. The Journal of Clinical Psychiatry, 69(2), 222-32. doi: 10.4088/JCP.v69n0208

Klein, D. N., Schwartz, J. E., Santiago, N. J., Vivian, D., Vocisano, C., Castonguay, L. G., ... \& Keller, M. B. (2003). Therapeutic alliance in depression treatment: controlling for prior change and patient characteristics. Journal of Consulting and Clinical Psychology, 71(6), 997-1006. doi: 10.1037/0022-006X.71.6.997

Kobayashi, M., \& Pascual-Leone, A. (2003). Transcranial magnetic stimulation in neurology. The Lancet Neurology, 2(3), 145-156.

Landa-Durán, P. (2009). Enseñanza de la psicología clínica conductual, presencial vs. En línea: la importancia del diseño instruccional. Acta Colombiana de Psicología, 12(1), 109-123. Recuperado de http://www.scielo.org.co/scielo. php?script=sci_arttext\&pid=S0123-91552009000100010

Larsen, S., \& Sherlin, L. (2013). Neurofeedback: an emerging technology for treating central nervous system dysregulation. The Psychiatric Clinics of North America, 36(1), 163168. doi: 10.1016/j.psc.2013.01.005

Leon-Sarmiento, F. E., Granadillo, E., \& Bayona, E. A. (2013). [Present and future of the transcranial magnetic stimulation]. Investigación Clínica, 54(1), 74-89. 
Lofthouse, N., Arnold, L. E., Hersch, S., Hurt, E., \& DeBeus, R. (2012). A review of neurofeedback treatment for pediatric ADHD. Journal of Attention Disorders, 16(5), 351-372. doi:10.1177/1087054711427530. doi: $10.1177 / 1087054711427530$

Loo, S. K., \& Makeig, S. (2012). Clinical utility of EEG in attention-deficit/hyperactivity disorder: a research update. Neurotherapeutics: The Journal of the American Society for Experimental NeuroTherapeutics, 9(3), 569-587. doi: $10.1007 / \mathrm{s} 13311-012-0131-\mathrm{z}$

Luyten, P., Blatt, S. J., Van Houdenhove, B. \& Corveleyn, J. (2006). Depression research and treatment: Are we skating to where the puck is going to be? Clinical Psychology Review, 26, 985-999.

Gonzalez-Lorenzo, M., Peñate, W., Pitti, C. T., Bethencourt, J., de la Fuente, J., \& Gracia, R. (2011). Efficacy of virtual reality exposure therapy combined with two pharmacotherapies in the treatment of agoraphobia. International Journal of Clinical and Health Psychology, 11, 189-203.

Meyerbroeker, K., Morina, N., Kerkhof, G. a, \& Emmelkamp, P. M. G. (2013). Virtual reality exposure therapy does not provide any additional value in agoraphobic patients: a randomized controlled trial. Psychotherapy and Psychosomatics, 82(3), 170-176. doi: 10.1159/000342715

Meyerbröker, K., \& Emmelkamp, P. M. G. (2010). Virtual reality exposure therapy in anxiety disorders: a systematic review of process-and-outcome studies. Depression and Anxiety, 27(10), 933-944. doi: 10.1002/da.20734

Montes González, J. A., \& Ochoa Angrino, S. (2006). Apropiación de las tecnologías de la información y comunicación en cursos universitarios. Acta Colombiana de Psicología, 9(2), 87-100. Recuperado de http://www.redalyc.org/ pdf/798/79890209.pdf

Moriyama, T. S., Polanczyk, G., Caye, A., Banaschewski, T., Brandeis, D., \& Rohde, L. A. (2012). Evidence-based information on the clinical use of neurofeedback for ADHD. Neurotherapeutics: The Journal of the American Society for Experimental NeuroTherapeutics, 9(3), 588-598. doi:10.1007/s13311-012-0136-7

Mustaca, A. (2003). Análisis experimental del comportamiento y neurociencias. Acta Colombiana de Psicología, 2, 7-22. Recuperado de http://www.redalyc.org/articulo. oa? id $=79801002$

Nelson, E.-L., Bui, T. N., \& Velasquez, S. E. (2011). Telepsychology: research and practice overview. Child and Adolescent Psychiatric Clinics of North America, 20(1), 67-79. doi: 10.1016/j.chc.2010.08.005

Newman, M. G., Szkodny, L. E., Llera, S. J., \& Przeworski, A. (2011). A review of technology-assisted self-help and minimal contact therapies for anxiety and depression: is human contact necessary for therapeutic efficacy? Clinical Psychology Review, 31(1), 89-103. doi: 10.1016/j. cpr.2010.09.008
Nickelson, D. W. (1998). Telehealth and the evolving health care system: Strategic opportunities for professional psychology. Research and Practice, 29, 527-535.

Niv, S. (2013). Clinical efficacy and potential mechanisms of neurofeedback. Personality and Individual Differences, 54(6), 676-686. doi: 10.1016/j.paid.2012.11.037

Norris, A.C. (2002). Essentials of telemedicine and telecare. New York: Wiley.

North, M., \& North, S. (1994). Virtual Environments and psychological disorders. Electronic Journal of Virtual Culture, 2, 37-42.

Obando, O. L., Villalobos, M. E., \& Arango, S. L. (2010). Resiliencia en niños con experiencias de abandono. Acta Colombiana de Psicología, 13(2), 149-159. Recuperado de http://portalweb.ucatolica.edu.co/easyWeb2/files/23_4917_ v12n3-art12.pdf

Opriş, D., Pintea, S., García-Palacios, A., Botella, C., Szamosközi, Ş., \& David, D. (2012). Virtual reality exposure therapy in anxiety disorders: a quantitative meta-analysis. Depression and Anxiety, 29(2), 85-93. doi: 10.1002/ da. 20910

Ossa, J. C. (2011). Funcionamiento cognitivo: un inextricable juego de pérdidas y ganancias. Acta Colombiana de Psicología, 14(2), 45-55. Recuperado de http://portalweb. ucatolica.edu.co/easyWeb2/files/23_7747_v14n2-art4.pdf

Peñate, W. (2012). About the effectiveness of telehealth procedures in psychological treatments. International Journal of Clinical and Health Psychology, 12, 475-487.

Peñate, W., Roca Sanchez, M.-J., Pitti Gonzalez, C. T., Manuel, J., Antonio, J., Fuente, D., \& Gracia, R. (2014). Cognitivebehavioral treatment and antidepressants combined with virtual reality exposure for patients with chronic agoraphobia. International Journal of Clinical and Health Psychology, 14, 330-338. doi: 10.1016/S1697-2600(14)70032-8

Pérez-Wehbe, A. I., Perestelo-Pérez, L., Bethencourt-Pérez, J. M., Cuellar-Pompa, L., \& Peñate, W. (2014). Treatmentresistant depression: a systematic review of systematic reviews. International Journal of Clinical and Health Psychology, 14, 145-155.

Roth, S. R., Sterman, M. B., \& Clemente, C. C. (1967). Comparison of EEG correlates of reinforcement, internal inhibition, and sleep. Electroencephalography and Clinical Neurophysiology, 23, 509-520.

Rossi, S., Hallett, M., Rossini, P. M., \& Pascual-Leone, A. (2009). Safety, ethical considerations, and application guidelines for the use of transcranial magnetic stimulation in clinical practice and research. Clinical Neurophysiology: Official Journal of the International Federation of Clinical Neurophysiology, 120(12), 2008-2039. doi: 10.1016/j. clinph.2009.08.016

Schutter, D. J. L. G. (2009) Antidepressant efficacy of high-frequency transcranial magnetic stimulation over the left dorsolateral prefrontal cortex in double-blind sham-controlled 
designs: A meta-analysis. Psychological Medicine, 39, 6575. doi: $10.1017 / \mathrm{S} 0033291708003462$

Shiban, Y., Pauli, P., \& Mühlberger, A. (2013). Effect of multiple context exposure on renewal in spider phobia. Behaviour Research and Therapy, 51, 68-74. doi: 10.1016/j. brat.2012.10.007

Sterman, M. B., \& Egner, T. (2006). Foundation and practice of neurofeedback for the treatment of epilepsy. Applied Psychophysiology and Biofeedback, 31(1), 21-35. doi: 10.1007/s10484-006-9002-x

Theodoroff, S. M., \& Folmer, R. L. (2013). Repetitive transcranial magnetic stimulation as a treatment for chronic tinnitus: a critical review. Otology \& Neurotology: Official Publication of the American Otological Society, American Neurotology Society [and] European Academy of Otology and Neurotology, 34(2), 199-208. doi: 10.1097/MAO.0b013e31827b4d46
Titov, N. (2007). Status of computerized cognitive behavioural therapy for adults. Australian and New Zealand Journal of Psychiatry, 41, 95-114. doi: 10.1080/00048670601109873

Vanni, F., Conversano, C., Del Debbio, A., Landi, P., Carlini, M., Fanciullacci, C., ... Dell'Osso, L. (2013). A survey on virtual environment applications to fear of public speaking. European Review for Medical and Pharmacological Sciences, 17(12), 1561-1568. Recuperado de http://www.europeanreview.org/wp/wp-content/uploads/1561-1568.pdf

Wilson, G. T. (1996). Manual-based treatments: the clinical application of research findings. Behaviour Research and Therapy, 34(4), 295-314. doi: 10.1016/0005-7967(95)00084-4. 